Pacific

Journal of

Mathematics

GROUPS OF LINEAR ISOMETRIES ON MULTIPLIER $C^{*}$-ALGEBRAS

Claudio D’Antoni and LÁszló Zsidó

Volume $193 \quad$ No. 2

April 2000 


\title{
GROUPS OF LINEAR ISOMETRIES ON MULTIPLIER C*-ALGEBRAS
}

\author{
Claudio D’Antoni And LÁsZló Zsidó
}

\begin{abstract}
For $A C^{*}$-algebra and $M(A)$ its multiplier algebra, the weak topologies $\sigma\left(M(A), A^{*}\right)$ and $\sigma\left(A^{*}, M(A)\right)$ are shown to have the Krein property, claiming the compactness of the closed convex hull of every compact set. This has relevant consequences concerning the analytic generator of strictly continuous one-parameter groups of strictly continuous linear operators on $M(A)$.

Furthermore, it is proved that there exists an one-to-one correspondence between surjective linear isometries on $A$ and strictly bicontinuous, surjective linear isometries on $M(A)$, as well as between strongly continuous respectively strictly continuous locally compact groups of them. In the case of connected groups, they all arise from $*$-automorphism groups by perturbation with a cocycle.
\end{abstract}

\section{Introduction.}

Recently the search for a sound $C^{*}$-algebraic framework for quantum groups has renewed the attention for analysis on multiplier $C^{*}$-algebras: The present setting requires a more precise understanding of groups of linear isometries on multiplier $C^{*}$-algebras. Of particular relevance seems to be the structure of the analytic generator in the one-parameter case (see e.g., [Kus]).

The multiplier algebra $M(A)$ of $C^{*}$-algebra $A$ is the non-commutative generalization of the Stone-Cech compactification $\beta \Omega$ of a locally compact topological space $\Omega$. In the commutative setting the canonical pairing between the bounded continuous functions on $\Omega$, identified with the continuous functions on $\beta \Omega$, and the bounded regular Borel measures on $\Omega$ has been intensively investigated (see e.g., $[\mathbf{C n w}]$ and $[\mathbf{H J}]$ ). In the non-commutative frame the analogous pairing between $M(A)$ and the dual $A^{*}$ is obtained from the natural duality between $A^{* *}$ and $A^{*}$ considering $M(A)$ embedded in $A^{* *}$. There exists a natural topology on $M(A)$, called the strict topology, which is compatible with this duality between $M(A)$ and $A^{*}$.

Our first goal is to prove in Section 1 that the corresponding weak topologies on $M(A)$ and $A^{*}$ have the so-called Krein property (indicated in $[\mathbf{C}-\mathbf{Z}]$ as 
axiom $A_{1}$ ), claiming the compactness of the closed convex hull of every compact set. This entails the Pettis integrability of the $\sigma\left(M(A), A^{*}\right)$-continuous $M(A)$-valued respectively $\sigma\left(A^{*}, M(A)\right)$-continuous $A^{*}$-valued functions and allows to apply the results from $[\mathbf{C}-\mathbf{Z}]$ and $[\mathbf{Z 1}]$ to the one-parameter operator groups on $M(A)$. We notice that for commutative $A$ the Krein property for $\sigma\left(M(A), A^{*}\right)$ and $\sigma\left(A^{*}, M(A)\right)$ is already known (see $[\mathbf{H J}]$ ).

Subsequently, in Section 2 we investigate the extendibility of bounded linear maps $\Phi: A \rightarrow B, A$ and $B C^{*}$-algebras, to strictly continuous linear maps $M(A) \rightarrow M(B)$. We prove that for $\Phi$ Jordan $*$-homomorphism, whose range generates $B$, this extension exists and it is the only Jordan *homomorphism $M(\Phi): M(A) \rightarrow M(B)$ extending $\Phi$. A similar result holds also for surjective linear isometries. Moreover, any strictly bicontinuous surjective linear isometry $M(A) \rightarrow M(B)$ maps $A$ onto $B$, hence it is an extension of a surjective linear isometry $A \rightarrow B$. For $A$ and $B$ separable, making use of a result by L.G. Brown (see $[\mathbf{B r}]$ ), we get the automatic strict bicontinuity of all surjective linear isometries $M(A) \rightarrow M(B)$. The same holds, for a different reason, if $A$ and $B$ are simple.

In Section 3 we study families $\left(\Phi_{t}\right)_{t}$ of linear isometries depending on a parameter $t$. We prove that continuous dependence in the strong operator topology of $\left(\Phi_{t}\right)_{t}$ goes in pointwise strictly continuous dependence of $M\left(\Phi_{t}\right)_{t}$. As a consequence, we get a one-to-one correspondence between the strongly continuous representations of a locally compact group $G$ by linear isometries on a $C^{*}$-algebra $A$ and the strictly continuous representations of $G$ by strictly bicontinuous (automatic, if $A$ separable or simple!) linear isometries on $M(A)$. In the one-parameter case the graph of the analytic generator of the extension turns out to be the strict closure of the graph of the analytic generator of the original group.

Using results due to R.V. Kadison (see $[\mathbf{K a d} 2]$ ) we prove also a structure theorem for strongly continuous representations of connected topological groups $G$ by linear isometries on a $C^{*}$-algebra $A$. Namely, they all arise from strongly continuous representations of $G$ by *-automorphisms of $A$, perturbing them with a cocycle. We notice that the $W^{*}$-algebra counterpart of this result holds only under additional assumptions, for example, assuming that the centre of the algebra is atomic.

Finally we show that representations of locally compact groups by linear isometries on separable $C^{*}$-algebras are strongly continuous under minimal regularity assumptions and they induce strongly continuous representations on separable, invariant $C^{*}$-subalgebras of the corona algebra.

\section{The Krein Property.}

A relevant property for a locally convex topological vector space $X$ is the following one: 
$(K)$ the closed convex hull of every weakly compact subset of $X$ is compact.

According to a well known result of M.G. Krein, V.L. Smulian and A. Grothendieck (see e.g., [Sch], Th. IV.11.4), the closed convex hull of a weakly compact subset of $X$ is weakly compact if (and only if) it is complete with respect to the associated Mackey topology. Therefore let us call $(K)$ the "Krein property".

We notice two consequences of the Krein property:

If $X$ has the Krein property then every weakly continuous map $\Omega \rightarrow X, \Omega$ compact topological space, is Pettis integrable with respect to any Radon measure on $\Omega$ (see e.g., [Rud], Th. 3.27, [Sch], Exercise IV.39 (a), [Ar], Prop. 1.2, [C-Z], Prop. 1.4).

A second consequence concerns "dual pairs of Banach spaces", that is pairs $(X, \mathcal{F})$ of Banach spaces together with a bilinear functional

$$
X \times \mathcal{F} \ni(x, \varphi) \mapsto\langle x, \varphi\rangle
$$

satisfying

$$
\begin{array}{ll}
\|x\|=\sup \{|\langle x, \varphi\rangle| ; \varphi \in \mathcal{F},\|\varphi\| \leq 1\}, & x \in X, \\
\|x\|=\sup \{|\langle x, \varphi\rangle| ; x \in \mathcal{F},\|x\| \leq 1\}, & \varphi \in \mathcal{F} .
\end{array}
$$

If $X$, endowed with the weak topology $\sigma(X, \mathcal{F})$, and $\mathcal{F}$, endowed with $\sigma(\mathcal{F}, X)$, have the Krein property then, for any $\sigma(X, \mathcal{F})$-continuous oneparameter group $t \mapsto U_{t}$ of $\sigma(X, \mathcal{F})$-continuous linear maps on $X$, the analytic extension operators $U_{z}, z \in \mathbb{C}$, are (besides being $\sigma(X, \mathcal{F})$-densely defined, a consequence of the Pettis integrability of $U) \sigma(X, \mathcal{F})$-closed (see [C-Z], Th. 2.4). Moreover, denoting by $U^{\mathcal{F}}$ the adjoint group on $\mathcal{F}, U_{z}^{\mathcal{F}}$ is the adjoint of $U_{z}$ in $\mathcal{F}$ (see [Z1], Th. 1.1).

If $\mathcal{F}=X^{*}$ then, according to the Krein theorem, the principle of uniform boundedness and the Alaoglu theorem, $\sigma(X, \mathcal{F})$ and $\sigma(\mathcal{F}, X)$ have the Krein property. We shall prove this for an other kind of dual pair of Banach spaces, in general not of the above form: We shall deal with dual pairs consisting of the multiplier algebra $M(A)$ and the dual space $A^{*}$ of a $C^{*}$-algebra $A$.

The multiplier algebra $M(A)$, first considered in [Buc] (for commutative $A$ ) and [Bus] (for general $C^{*}$-algebra $A$ ), is (*-isomorphic to) the $C^{*}$ subalgebra

$$
\left\{x \in A^{* *} ; x a, a x \in A \text { for all } a \in A\right\}
$$

of the second dual $A^{* *}$. For its basic theory we send to [Ped1], 3.12 and [WO], Chapter 2.

The canonical duality between $A^{* *}$ and $A^{*}$ induces a pairing

$$
M(A) \times A^{*} \ni(x, \varphi) \mapsto\langle x, \varphi\rangle
$$

which makes $\left(M(A), A^{*}\right)$ a dual pair of Banach spaces. We recall that the strict topology $\beta$ on $M(A)$ is the locally convex vector space topology defined 
by the seminorms

$$
x \mapsto\|x a\| \text { and } x \mapsto\|a x\|, \quad a \in A .
$$

It is complete and compatible with the duality between $M(A)$ and $A^{*}$ (see [T1], Cor. 2.3). Hence the strict topology is weaker that the norm-topology on $M(A)$, but stronger than the restriction to $M(A)$ of the weak * topology of $A^{* *}$. $A$ is a strictly dense, norm-closed two-sided ideal of $M(A)$. More precisely, if $\left(u_{\iota}\right)_{\iota}$ is any two-sided approximate unit for $A$ then $u_{\iota} \rightarrow 1_{A^{* *}} \in$ $M(A)$ strictly.

The goal of this section is to prove:

Theorem 1.1 (on the Krein property for multipliers). Let $A$ be an arbitrary $C^{*}$-algebra. Then $M(A)$ with $\sigma\left(M(A), A^{*}\right)$ and $A^{*}$ with $\sigma\left(A^{*}, M(A)\right)$ have the Krein property.

Since the strict topology is complete, also the Mackey topology $\tau\left(M(A), A^{*}\right)$ is complete (see e.g., [B], IV.5, Remark 2). Therefore the Krein property of $\sigma\left(M(A), A^{*}\right)$ follows directly from the Krein theorem.

The proof of the Krein property of $\sigma\left(A^{*}, M(A)\right)$ is more involved. Let us shortly comment the already known results concerning it. We notice that the Krein property for $\sigma\left(A^{*}, M(A)\right)$ is equivalent to saying that all seminorms

$$
M(A) \in x \mapsto \sup \{|\langle x, \varphi\rangle| ; \varphi \in \mathcal{K}\}
$$

with $\mathcal{K} \subset A^{*} \sigma\left(A^{*}, M(A)\right)$-compact are $\tau\left(M(A), A^{*}\right)$-continuous, that is to the $\tau\left(M(A), A^{*}\right)$-equicontinuity of any $\sigma\left(A^{*}, M(A)\right)$-compact subset of $A^{*}$.

If $\tau$ is a locally convex vector space topology on $M(A)$, compatible with the duality between $M(A)$ and $A^{*}$, then $M(A), \tau$ is usually called strong Mackey space whenever any $\sigma\left(A^{*}, M(A)\right)$-compact subset of $A^{*}$ is $\tau$-equicontinuous. In other words, $M(A), \tau$ is strong Mackey if and only if $\tau=$ $\tau\left(M(A), A^{*}\right)$ and $\sigma\left(A^{*}, M(A)\right)$ has the Krein property.

There are criteria in order that the strict topology $\beta$ on $M(A)$ be strong Mackey: This happens for commutative $A$ whenever the Gelfand spectrum of $A$ is paracompact ([Cnw], Th. 2.6) and, more generally, for arbitrary $A$, whenever $A$ has a "well behaved" approximate unit ([T2], Cor. 3.4). In particular, in this case $\sigma\left(A^{*}, M(A)\right)$ has the Krein property. However, even for commutative $A, \beta$ is not always equal to $\tau\left(M(A) A^{*}\right)$, so $M(A), \beta$ is not always strong Mackey space (see [Cnw], Remarks on p. 481). Nevertheless, it was proved that $\sigma\left(A^{*}, M(A)\right)$ has the Krein property for any commutative $A$ (see $[\mathbf{H J}]$, Th. 2). Actually this proof inspired our proof of the Krein property of $\sigma\left(A^{*}, M(A)\right)$ for general $A$.

The main ingredient in proving the Krein property of $\sigma\left(A^{*}, M(A)\right)$ is the following convergence result: 
Lemma 1.2. Let be $A$ a $C^{*}$-algebra, $\mathcal{K}$ a $\sigma\left(A^{*}, M(A)\right)$-compact subset of $A_{h}^{*}=\left\{\varphi \in A^{*} ; \varphi=\varphi^{*}\right\}$, and $\mu$ a positive Radon measure on the compact topological space $\mathcal{K}, \sigma\left(A^{*}, M(A)\right)$. Then, for any (increasing, positive) approximate unit $\left(u_{\iota}\right)_{\iota}$ of $A$ and any $x \in M(A)$,

$$
\int_{\mathcal{K}}\left\langle x^{*} u_{\iota} x, \varphi\right\rangle d \mu(\varphi) \stackrel{\iota}{\rightarrow} \int_{\mathcal{K}}\left\langle x^{*} x, \varphi\right\rangle d \mu(\varphi) .
$$

We notice that if $\mathcal{K}$ would be contained in $A_{+}^{*}$ then, according to the Dini theorem, we would have $\left\langle x^{*} u_{\iota} x, \varphi\right\rangle \stackrel{\iota}{\rightarrow}\left\langle x^{*} x, \varphi\right\rangle$ uniformly for $\varphi \in \mathcal{K}$ and the statement of the Lemma would follow trivially.

Proof. First we consider the case $x=1_{A^{* *}}$.

Let us define the lower $\sigma\left(A^{*}, M(A)\right)$-semicontinuous functions $g_{\iota}: \mathcal{K} \rightarrow \mathbb{R}$ by

$$
g_{\iota}(\varphi)=\sup \left\{|\langle a, \varphi\rangle| ; a^{*}=a \in A,-u_{\iota} \leq a \leq u_{\iota}\right\} \leq\|\varphi\| .
$$

Then $\left(g_{\iota}\right)_{\iota}$ is upward directed and pointwise convergent to $\mathcal{K} \in \varphi \mapsto\|\varphi\|$, so

$$
\int_{\mathcal{K}} g_{\iota}(\varphi) d \mu(\varphi) \stackrel{\iota}{\rightarrow} \int_{\mathcal{K}}\|\varphi\| d \mu(\varphi) .
$$

For let $\varphi \in \mathcal{K}$ and $\varepsilon>0$ be arbitrary. Choosing $a^{*}=a \in A,\|a\| \leq 1$, with

$$
|\langle a, \varphi\rangle| \geq\|\varphi\|-\frac{\varepsilon}{2}
$$

we have

$$
\left(u_{\iota}^{1 / 2} a u_{\iota}^{1 / 2}\right)^{*}=u_{\iota}^{1 / 2} a u_{\iota}^{1 / 2} \in A,
$$

so

$$
\begin{aligned}
g_{\iota}(\varphi) & \geq\left|\left\langle u_{\iota}^{1 / 2} a u_{\iota}^{1 / 2}, \varphi\right\rangle\right| \\
& \geq|\langle a, \varphi\rangle|-\left|\left\langle a-u_{\iota}^{1 / 2} a u_{\iota}^{1 / 2}, \varphi\right\rangle\right| \\
& \geq\|\varphi\|-\frac{\varepsilon}{2}-\|\varphi\| \cdot\left\|a-u_{\iota}^{1 / 2} a u_{\iota}^{1 / 2}\right\| .
\end{aligned}
$$

Since

$$
\begin{aligned}
\left\|a-u_{\iota}^{1 / 2} a u_{\iota}^{1 / 2}\right\| & \leq\left\|\left(1_{A^{* *}}-u_{\iota}^{1 / 2}\right) a\right\|+\left\|u_{\iota}^{1 / 2} a\left(1_{A^{* *}}-u_{\iota}^{1 / 2}\right)\right\| \\
& \leq 2 \cdot\left\|a\left(1_{A^{* *}}-u_{\iota}^{1 / 2}\right)^{2} a\right\|^{1 / 2} \\
& \leq 2 \cdot\left\|a\left(1_{A^{* *}}-u_{\iota}\right) a\right\|^{1 / 2} \\
& \leq 2 \cdot\left\|a-u_{\iota} a\right\|^{1 / 2}
\end{aligned}
$$

and $\left(u_{\iota}\right)_{\iota}$ is an approximate unit for $A$, it follows the existence of some $\iota_{\varepsilon}$ such that

$$
g_{\iota}(\varphi) \geq\|\varphi\|-\varepsilon \text { for all } \iota \geq \iota_{\varepsilon}
$$


On the other hand,

$$
\left|\left\langle 1_{A^{* *}}-u_{\iota}, \varphi\right\rangle\right| \leq\|\varphi\|-g_{\iota}(\varphi) \text { for all } \varphi \in \mathcal{K} \text { and } \iota .
$$

Indeed, for every $a^{*}=a \in A$ with $-u_{\iota} \leq a \leq u_{\iota}$,

$$
\begin{aligned}
|\langle a, \varphi\rangle| & \leq\left|\left\langle a, \varphi_{+}\right\rangle\right|+\left|\left\langle a, \varphi_{-}\right\rangle\right| \\
& \leq\left\langle u_{\iota}, \varphi_{+}\right\rangle+\left\langle u_{\iota}, \varphi_{-}\right\rangle \\
& =\left\langle u_{\iota},|\varphi|\right\rangle, \\
\left|\left\langle 1_{A^{* *}}-u_{\iota}, \varphi\right\rangle\right|+|\langle a, \varphi\rangle| & \leq\left\langle 1_{A^{* *}}-u_{\iota},|\varphi|\right\rangle+\left\langle u_{\iota},|\varphi|\right\rangle \\
& =\left\langle 1_{A^{* *}},|\varphi|\right\rangle \\
& =\|\varphi\|,
\end{aligned}
$$

hence

$$
\left|\left\langle 1_{A^{* *}}-u_{\iota}, \varphi\right\rangle\right|+g_{\iota}(\varphi) \leq\|\varphi\| .
$$

Therefore

$$
\begin{aligned}
& \left|\int_{\mathcal{K}}\left\langle 1_{A^{* *}}, \varphi\right\rangle d \mu(\varphi)-\int_{\mathcal{K}}\left\langle u_{\iota}, \varphi\right\rangle d \mu(\varphi)\right| \\
& \leq \int_{\mathcal{K}}\left|\left\langle 1_{A^{* *}}-u_{\iota}, \varphi\right\rangle\right| d \mu(\varphi) \\
& \leq \int_{\mathcal{K}}\left(\|\varphi\|-g_{\iota}(\varphi)\right) d \mu(\varphi)
\end{aligned}
$$

and (1.1) yields

$$
\int_{\mathcal{K}}\left\langle u_{\iota}, \varphi\right\rangle d \mu(\varphi) \stackrel{\iota}{\rightarrow} \int_{\mathcal{K}}\left\langle 1_{A^{* *}}, \varphi\right\rangle d \mu(\varphi) .
$$

Now let $x \in M(A)$ be arbitrary.

The map

$$
\Phi_{x}: A_{h}^{*} \ni \varphi \mapsto \varphi\left(x^{*} \cdot x\right) \in A_{h}^{*}
$$

is $\sigma\left(A^{*}, M(A)\right)$-continuous, so $\mathcal{K}_{x}=\Phi_{x}(\mathcal{K})$ is a $\sigma\left(A^{*}, M(A)\right)$-compact subset of $A_{h}^{*}$. Denoting by $\mu_{x}$ the image of $\mu$ under $\Phi, \mu_{x}$ is a positive Radon measure on $\mathcal{K}_{x}, \sigma\left(A^{*}, M(A)\right)$ and

$$
\int_{\mathcal{K}_{x}} f(\psi) d \mu_{x}(\psi)=\int_{\mathcal{K}} f\left(\Phi_{x}(\varphi)\right) d \mu(\varphi)
$$

for all $\sigma\left(A^{*}, M(A)\right)$-continuous $f: \mathcal{K}_{x} \rightarrow \mathbb{R}$. Using the above equality with $f(\psi)=\left\langle u_{\iota}, \psi\right\rangle$ respectively $f(\psi)=\left\langle 1_{A^{* *}}, \psi\right\rangle$ and applying the first part of the proof to $\mathcal{K}_{x}, \mu_{x}$, we get

$$
\begin{aligned}
& \int_{\mathcal{K}}\left\langle x^{*} u_{\iota} x, \varphi\right\rangle d \mu(\varphi) \\
& =\int_{\mathcal{K}}\left\langle u_{\iota}, \psi\right\rangle d \mu_{x}(\psi) \stackrel{\iota}{\rightarrow} \int_{\mathcal{K}_{x}}\left\langle 1_{A^{* *}}, \psi\right\rangle d \mu_{x}(\psi)
\end{aligned}
$$




$$
=\int_{\mathcal{K}}\left\langle x^{*} x, \varphi\right\rangle d \mu(\varphi)
$$

Proof of the theorem. We have already seen that the completeness of the strict topology on $M(A)$ implies the Krein property for $\sigma\left(M(A), A^{*}\right)$.

The Krein property for $\sigma\left(A^{*}, M(A)\right)$ means that every $\sigma\left(A^{*}, M(A)\right)$ compact $\mathcal{K} \subset A^{*}$ is contained in a $\sigma\left(A^{*}, M(A)\right)$-compact convex subset of $A^{*}$. Let us first consider the case of a $\sigma\left(A^{*}, M(A)\right)$-compact $\mathcal{K} \subset A_{h}^{*}$.

Let $P(\mathcal{K})$ denote the weak* compact convex set of all probability Radon measures on $\mathcal{K}$, endowed with $\sigma\left(A^{*}, M(A)\right)$. For every $\mu \in P(\mathcal{K})$ we can define $\Phi(\mu) \in A^{*}$ by putting

$$
\langle a, \Phi(\mu)\rangle=\int_{\mathcal{K}}\langle a, \varphi\rangle d \mu(\varphi), \quad a \in A .
$$

Then actually holds:

$$
\langle x, \Phi(\mu)\rangle=\int_{\mathcal{K}}\langle x, \varphi\rangle d \mu(\varphi), \quad x \in M(A)
$$

(in other words there exists the Pettis integral $\sigma\left(A^{*}, M(A)\right)-\int_{\mathcal{K}} \varphi d \mu(\varphi) \in$ $\left.A^{*}\right)$. Indeed, choosing an approximate unit $\left(u_{\iota}\right)_{\iota}$ of $A$ and applying the above lemma, we have for every $0 \leq x \in M(A)$

$$
\left\langle x^{1 / 2} u_{\iota} x^{1 / 2}, \Phi(\mu)\right\rangle=\int_{\mathcal{K}}\left\langle x^{1 / 2} u_{\iota} x^{1 / 2}, \varphi\right\rangle d \mu(\varphi) \stackrel{\iota}{\rightarrow} \int_{\mathcal{K}}\langle x, \varphi\rangle d \mu(\varphi) .
$$

But on the other hand, since $x^{1 / 2} u_{\iota} x^{1 / 2} \rightarrow x$ in the weak* topology of $A^{* *}$, we have also

$$
\left\langle x^{1 / 2} u_{\iota} x^{1 / 2}, \Phi(\mu)\right\rangle \stackrel{\iota}{\rightarrow}\langle x, \Phi(\mu)\rangle .
$$

By (1.2) the affine map $\Phi: P(\mathcal{K}) \rightarrow A^{*}$ is continuous with respect to the weak* topology on $P(\mathcal{K})$ and $\sigma\left(A^{*}, M(A)\right)$ on $A^{*}$. Consequently $\Phi P(\mathcal{K})$ is a $\sigma\left(A^{*}, M(A)\right)$-compact convex subset of $A^{*}$ containing

$$
\left\{\Phi\left(\delta_{\varphi}\right) ; \delta_{\varphi} \text { the Dirac measure in } \varphi \in \mathcal{K}\right\}=\mathcal{K} .
$$

Now let the $\sigma\left(A^{*}, M(A)\right)$-compact set $\mathcal{K} \subset A^{*}$ be arbitrary. Since $A^{*} \ni$ $\varphi \mapsto \varphi^{*} \in A^{*}$ is $\sigma\left(A^{*}, M(A)\right)$-continuous, $\operatorname{Re} \mathcal{K}, \operatorname{Im} \mathcal{K} \subset A_{h}$ are also $\sigma\left(A^{*}, M(A)\right)$-compact. According to the above part of the proof, there are $\sigma\left(A^{*}, M(A)\right)$-compact convex sets $\operatorname{Re} \mathcal{K} \subset \mathcal{K}_{1} \subset A^{*}$ and $\operatorname{Im} \mathcal{K} \subset \mathcal{K}_{2} \subset A^{*}$. Then $\mathcal{K}_{1}+i \mathcal{K}_{2}$ is a $\sigma\left(A^{*}, M(A)\right)$-compact convex subset of $A^{*}$ containing $\mathcal{K}$.

By the above theorem the results from $[\mathbf{C}-\mathbf{Z}]$ and $[\mathbf{Z 1}]$ are available for $\sigma\left(M(A), A^{*}\right)$-continuous one-parameter groups of $\sigma\left(M(A), A^{*}\right)$-continuous linear operators on $M(A)$. In particular, [C-Z], Th. 2.4 and $[\mathbf{Z 1}]$, Th. 1.1 yield: 
Corollary. Let $A$ be a $C^{*}$-algebra, $t \rightarrow \alpha_{t}$ a $\sigma\left(M(A), A^{*}\right)$-continuous oneparameter group of $\sigma\left(M(A), A^{*}\right)$-continuous linear operators on $M(A), t \mapsto$ $\alpha_{t}^{*}$ the adjoint group on $A^{*}$, and $z \in \mathbb{C}$. Define the linear operator $\alpha_{z}$ in $M(A)$ by putting $(x, y) \in$ graph $\left(\alpha_{z}\right)$ whenever $\mathbb{R} \ni t \mapsto \alpha_{t}(x) \in M(A)$ has a $\sigma\left(M(A), A^{*}\right)$-continuous extension on the closed horizontal strip between 0 and $\operatorname{Im} z$, which is analytic in the interior and takes the value $y$ in $z$. The linear operator $\alpha_{z}^{*}$ in $A^{*}$ is defined similarly. Then $\alpha_{z}$ is strictly densely defined, its graph is closed with respect to the product of the strict topologies on $M(A) \times M(A)$, and its adjoint in $A^{*}$ is $\alpha_{z}^{*}$.

\section{Jordan $*$-homomorphisms and linear isometries between multiplier algebras.}

In this section we investigate the extendibility of Jordan $*$-homomorphisms and linear isometries between $C^{*}$-algebras to similar maps between the respective multiplier algebras. We also describe those linear isometries between multiplier algebras, which arise as extensions.

Let $A, B$ be $C^{*}$-algebras. $\pi: A \rightarrow B$ is called Jordan $*$-homomorphism if it is a linear $*$-map satisfying

$$
\pi\left(x^{2}\right)=\pi(x)^{2}, \quad x \in A .
$$

It is well known that then (see e.g., $[\mathbf{S}-\mathbf{Z}], 6.6$ )

$$
\begin{gathered}
\pi(x y+y x)=\pi(x) \pi(y)+\pi(y) \pi(x), \\
\pi(x y x)=\pi(x) \pi(y) \pi(x), \\
x y=y x \Rightarrow \pi(x y)=\pi(x) \pi(y)
\end{gathered}
$$

with $x, y$ elements of $A$. The last statement implies immediately that if $A$ is unital then $\pi\left(1_{A}\right)$ is unit for the hereditary $C^{*}$-subalgebra $\operatorname{Her}_{B}(\pi(A)) \subset B$ generated by $\pi(A)$. In particular, $\pi$ being positive, $\|\pi\|=\left\|\pi\left(1_{A}\right)\right\|=1$ or 0 . For arbitrary $A$, the positive map $\pi$ being bounded, we can consider the Jordan $*$-homomorphism $\pi^{* *}: A^{* *} \rightarrow B^{* *}$ and the above remarks yields $\|\pi\|=\left\|\pi^{* *}\right\|=1$ or 0 .

Let $\pi: A \rightarrow B$ be a Jordan $*$-homomorphism. Then

$$
\|\pi(x) \pi(y)\| \leq\|x y\|, \quad x, y \in A_{h},
$$

where $A_{h}$ denotes the Hermitian part $\left\{a \in A ; a=a^{*}\right\}$ of $A$. Indeed,

$$
\begin{aligned}
\|\pi(x) \pi(y)\|^{2} & =\left\|\pi(y) \pi(x)^{2} \pi(y)\right\|=\left\|\pi\left(y x^{2} y\right)\right\| \\
& \leq\left\|y x^{2} y\right\|=\|x y\|^{2} .
\end{aligned}
$$

Furthermore,

$$
\pi(x)^{*} \pi(x) \leq \pi\left(x^{*} x+x x^{*}\right), \quad x \in A .
$$

For we just have to notice that

$$
\pi(x)^{*} \pi(x) \leq \pi(x)^{*} \pi(x)+\pi(x) \pi(x)^{*}=\pi\left(x^{*} x+x x^{*}\right) .
$$


It follows that

$$
\left\{\begin{array}{l}
J \subset B \text { norm closed two-sided ideal } \Rightarrow \\
\pi^{-1}(J) \subset A \text { norm closed two-sided ideal. }
\end{array}\right.
$$

Indeed, for any $a^{*}=a \in \pi^{-1}(J)$ and $x^{*}=x \in A$ we have by (2.2)

$$
\begin{aligned}
\pi(a x)^{*} \pi(a x) & \leq \pi\left((a x)^{*} a x+a x(a x)^{*}\right) \\
& =\pi\left(x a^{2} x+a x^{2} a\right) \\
& =\pi(x) \pi(a)^{2} \pi(x)+\pi(a) \pi(x)^{2} \pi(a) \in J,
\end{aligned}
$$

so, according to [Ped1], Prop. 1.4.5,

$$
\pi(a x) \in J, \text { i.e., } a x \in \pi^{-1}(J) .
$$

In particular, Ker $\pi=\pi^{-1}(\{0\})$ is a norm closed two-sided ideal in $A$. Thus $\pi$ factorizes in

$$
A \rightarrow A / \operatorname{Ker} \pi \stackrel{\tilde{\pi}}{\rightarrow} B
$$

where the first arrow denotes the quotient $*$-homomorphism and $\tilde{\pi}$ is an injective Jordan*-homomorphism. If $\pi$ is surjective then $\tilde{\pi}$ is Jordan *isomorphism, hence isometrical. Therefore in this case

$$
\|\pi(x)\|^{2}=\left\|\pi\left(x^{*} x\right)\right\|, \quad x \in A .
$$

The next result extends $[\mathbf{P}-\mathbf{S}]$, Th. 2 and $[\mathbf{P e d} 2]$, Th. 10 to Jordan *-homomorphisms between $C^{*}$-algebras. We recall that a $C^{*}$-algebra is called $\sigma$-unital whenever it contains a strictly positive element or, equivalently, it has a countable approximate unit (see [Ped1], 3.10.4, 3.10.5).

Proposition 2.1. Let $A, B$ be $C^{*}$-algebras, and $\pi: A \rightarrow B$ a Jordan *homomorphism with $\operatorname{Her}_{B}(\pi(A))=B$. Then

(i) there exists a unique extension of $\pi$ to a Jordan *-homomorphism $M(\pi): M(A) \rightarrow M(B)$, namely $M(\pi)=\pi^{* *} \mid M(A)$, which is strictly continuous and unital, hence carrying two-sided approximate units of $A$ in two-sided approximate units of $B$;

(ii) assuming that $A$ is $\sigma$-unital, $M(\pi)$ is surjective whenever $\pi$ is surjective.

Proof. (i) Let us first prove that if $A \subset A_{0} \subset A^{* *}$ is a $C^{*}$-algebra, $\rho: A_{0} \rightarrow$ $B^{* *}$ is a Jordan $*$-homomorphism extending $\pi$ and $\left(x_{\iota}\right)_{\iota}$ is a norm bounded net in $\left(A_{0}\right)_{h}$ with $\left\|x_{\iota} a\right\| \stackrel{\iota}{\rightarrow} 0$ for all $a \in A$, then $\left\|\rho\left(x_{\iota}\right) b\right\| \stackrel{\iota}{\rightarrow} 0$ for all $b \in B$. Indeed, applying (2.1) to $\rho$, we have for every $a \in A_{h}$

$$
\left\|\rho\left(x_{\iota}\right) \pi(a)\right\|=\left\|\rho\left(x_{\iota}\right) \rho(a)\right\| \leq\left\|x_{\iota} a\right\| \stackrel{\iota}{\rightarrow} 0 .
$$

Therefore the hereditary $C^{*}$-subalgebra

$$
\left\{b \in B ;\left\|\rho\left(x_{\iota}\right) b\right\| \stackrel{\iota}{\rightarrow} 0 \text { and }\left\|b \rho\left(x_{\iota}\right)\right\| \stackrel{\iota}{\rightarrow} 0\right\} \subset B \text { contains } \pi(A) .
$$


Now let $x^{*}=x \in M(A)$ be arbitrary. Choosing a norm bounded net $\left(a_{\iota}\right)_{\iota}$ in $A_{h}$ such that $a_{\iota} \stackrel{\iota}{\rightarrow} \rightarrow x$ strictly, by the above proved statement $\pi^{* *}(x) b=$ norm $-\lim _{\iota} \pi\left(a_{\iota}\right) b \in B$ for all $b \in B$, hence $\pi^{* *}(x) \in M(B)$. Thus $\pi^{* *} M(A) \subset M(B)$.

Again by the first part of the proof, any Jordan $*$-homomorphism $\rho$ : $M(A) \rightarrow M(B)$ extending $\pi$, in particular $M(\pi)=\pi^{* *} \mid M(A)$, is strictly continuous on every norm bounded subset of $M(A)$. It follows that $M(\pi)$ is the only such $\rho$. Moreover, since the strict topology $\beta$ on $M(A)$ is the finest locally convex vector space topology on $M(A)$ that agrees with $\beta$ on the norm bounded subsets of $M(A)$ (see [T1], Cor. 2.7), $M(\pi)$ is actually strictly continuous.

On the other hand, $\pi^{* *}\left(1_{A^{* *}}\right)$ is unit for $\operatorname{Her}_{B^{* *}}\left(\pi^{* *}\left(A^{* *}\right)\right) \supset \operatorname{Her}_{B}(\pi(A))$ $=B$, hence also for its weak* closure in $B^{* *}$ :

$$
\pi^{* *}\left(1_{A^{* *}}\right)=1_{B^{* *}} .
$$

In other words, $M(\pi)$ is unital.

(ii) Let

$$
A \stackrel{\pi_{0}}{\rightarrow} A / \operatorname{Ker} \pi \stackrel{\tilde{\pi}}{\rightarrow} B
$$

be the canonical factorization of $\pi$. By Pedersen's Tietze type extension theorem (see [Ped2], Th. 10 or [WO], Th. 2.3.9), $M\left(\pi_{0}\right)$ is surjective. On the other hand, $\tilde{\pi}$ being Jordan $*$-isomorphism, $M(\tilde{\pi})$ is Jordan $*$-isomorphism. We conclude that $M(\pi)=M(\tilde{\pi}) M\left(\pi_{0}\right)$ is surjective.

Now we prove a partial converse to Proposition 2.1:

Proposition 2.2. Let $A, B$ be $C^{*}$-algebras. Then any strictly bicontinuous Jordan $*$-isomorphism $\rho: M(A) \rightarrow M(B)$ maps $A$ onto $B$, so $\rho=M(\rho \mid A$ : $A \rightarrow B$ ). If $A$ and $B$ are both separable or both (topologically) simple, then we have this for any Jordan $*$-isomorphism $\rho: M(A) \rightarrow M(B)$, whose strict bicontinuity is hence automatical.

Proof. Let $\left(u_{\iota}\right)_{\iota}$ and $\left(v_{k}\right)_{k}$ be increasing, positive approximate units for $A$ respectively $B$. Since $A$ is two-sided ideal in $M(A)$ and $\rho^{-1}$ is strictly continuous,

$$
A \ni w_{\iota, k}=u_{\iota} \rho^{-1}\left(v_{k}\right) u_{\iota} \stackrel{\iota, k}{\rightarrow} 1_{A^{* *}} \quad \text { strictly. }
$$

In other words $\left(w_{\iota, k}\right)_{\iota, k}$ is a two-sided approximate unit for $A$. It follows for every $0 \leq a \in A$

$$
\begin{gathered}
\left\|a-a^{1 / 2} w_{\iota, k} a^{1 / 2}\right\| \stackrel{\iota, k}{\rightarrow} 0, \\
\left\|\rho(a)-\rho\left(a^{1 / 2} w_{\iota, k} a^{1 / 2}\right)\right\| \stackrel{\iota, k}{\rightarrow} 0,
\end{gathered}
$$

so, having

$$
\rho\left(a^{1 / 2} w_{\iota, k} a^{1 / 2}\right)=\rho\left(a^{1 / 2}\right) \rho\left(u_{\iota}\right) v_{k} \rho\left(u_{\iota}\right) \rho\left(a^{1 / 2}\right) \in B
$$


for all $\iota$ and $k, \rho(a) \in B$. Consequently

$$
\rho(A) \subset B .
$$

We get similarly also

$$
\rho^{-1}(B) \subset A, \text { i.e., } \rho(A) \supset B .
$$

Now let us assume that $A$ and $B$ are separable and $\rho: M(A) \rightarrow M(B)$ is an arbitrary Jordan $*$-isomorphism. By $[\mathbf{B r}]$, Cor. 6 (see also the remarks after Corollary 1.4 in $[\mathbf{D}-\mathbf{Z}]) A$ is the largest separable, norm closed, twosided ideal of $M(A)$, so (2.3) implies that $\rho(A)$ is the largest separable, norm closed, two-sided ideal of $M(B)$. Using again [Br], Cor. 6 , we conclude that $\rho(A)=B$.

Assuming finally that $A$ and $B$ are simple and nonzero, we can argue as above, using the fact that $A$ is the smallest nonzero, closed, two-sided ideal of $M(A)$, and similarly for $B$. Indeed, is $J$ is any nonzero, closed, two-sided ideal of $M(A)$, then the essentialness of $A$ yields

$$
A \cap J \supset A J \neq\{0\}
$$

and it follows by the simplicity of $A$ that $A \cap J=A$.

Now let $\Phi: A \rightarrow B$ be an isometrical linear bijection between $C^{*}$-algebras. Then there exist $\pi: A \rightarrow B$ Jordan $*$-isomorphism and $u \in M(B)$ unitary such that

$$
\Phi(x)=u \pi(x), \quad x \in A
$$

(see $[\mathbf{K a d} 1]$ for the case of unital $C^{*}$-algebras and $[\mathbf{P}-\mathbf{S}]$, Th. 1 for the general case). We notice that $u$, hence also $\pi$, is uniquely determined by $\Phi$ : according to Proposition 2.1., for any two-sided approximate unit $\left(u_{\iota}\right)_{\iota}$ of $A$ we have

$$
\pi\left(u_{\iota}\right) \rightarrow 1_{B^{* *}} \text { strictly, }
$$

so

$$
\Phi\left(u_{\iota}\right) \rightarrow u \text { strictly. }
$$

We call (2.4) the Kadison decomposition of $\Phi$.

The following theorem is the main result of this section (cf. with $[\mathbf{P}-\mathbf{S}]$, Th. 2):

Theorem 2.3 (on extension of linear isometries). Let $A, B$ be $C^{*}$-algebras. Then every isometric linear bijection $\Phi: A \rightarrow B$ has a unique extension to an isometric linear bijection $M(\Phi): M(A) \rightarrow M(B)$, which is strictly bicontinuous. Conversely, every strictly bicontinuous, isometrical linear bijection between $M(A)$ and $M(B)$ is of the above form. Moreover, if $A$ and $B$ are both separable or both (topologically) simple then the strict bicontinuity of any isometric linear bijection between $M(A)$ and $M(B)$ is automatical. 
Proof. Let $\Phi: A \rightarrow B$ be an isometric linear bijection, and $\Phi=u \pi$ its Kadison decomposition. Proposition 2.1 entails that, letting $M(\Phi)=$ $u M(\pi), M(\Phi)$ is a strictly bicontinuous, isometrical linear map of $M(A)$ onto $M(B)$. If $\Psi: M(A) \rightarrow M(B)$ is any isometric linear bijection extending $\Phi$ and $\Psi=v \rho$ is its Kadison decomposition, then $\Psi(A)=\Phi(A)=B$ implies $\rho(A)=v^{*} \Psi(A)=B$, so $\rho \mid A: A \rightarrow B$ is a Jordan $*$-isomorphism. The uniqueness of the Kadison decomposition of $\Phi=\Psi \mid A$ yields

$$
\pi=\rho \mid A, \quad u=v .
$$

But, again by Proposition 2.1, we have then $M(\pi)=\rho$ and it follows

$$
M(\Phi)=u M(\pi)=v \rho=\Psi .
$$

The second and the third statement of the theorem follow by taking the Kadison decomposition and applying Proposition 2.2 to the Jordan *-isomorphism in the decomposition.

We notice that every isometric linear bijection $\Phi: A \rightarrow B$ between $C^{*}$ algebras induces also an isometric linear bijection $C(\Phi): C(A) \rightarrow C(B)$ between the corresponding corona algebras $C(A)=M(A) / A$ and $C(B)=$ $M(B) / B: C(\Phi)$ carries the canonical image of $x \in M(A)$ in $C(A)$ in the canoncal image of $M(\Phi)(x)$ in $C(B)$.

\section{Groups of linear isometries on multiplier algebras.}

After having established in the preceeding section that surjective linear isometries between $C^{*}$-algebras and strictly bicontinuous surjective linear isometries between multiplier $C^{*}$-algebras are in one-to-one correspondence, let us now investigate the interplay of the continuity and analyticity properties of groups of such maps. We also investigate the structure of strongly continuous representations of connected groups by linear isometries on $C^{*}$ algebras.

Let $A, B$ be $C^{*}$-algebras, and $\pi: A \rightarrow B$ a Jordan *-homomorphism. Then the following variant of (2.1) holds:

$$
\left\{\begin{array}{l}
\|\pi(x) \pi(y)\| \leq\left(\|x y\|^{2}+\|y x\|^{2}\right)^{1 / 2} \leq\|x y\|+\|y x\| \\
\text { for all } x, y \in A, \text { one of which is self-adjoint. }
\end{array}\right.
$$

Indeed, let us assume, for example, that $y \in A_{h}$. By (2.2) we have $\pi(x)^{*} \pi(x)$ $\leq \pi\left(x^{*} x+x x^{*}\right)$, so

$$
\begin{aligned}
\|\pi(x) \pi(y)\|^{2} & =\left\|\pi(y) \pi(x)^{*} \pi(x) \pi(y)\right\| \\
& \leq\left\|\pi(y) \pi\left(x^{*} x+x x^{*}\right) \pi(y)\right\| \\
& =\left\|\pi\left(y\left(x^{*} x+x x^{*}\right) y\right)\right\| \\
& \leq\left\|y x^{*} x y+y x x^{*} y\right\|
\end{aligned}
$$




$$
\leq\|x y\|^{2}+\|y x\|^{2}
$$

First we prove:

Lemma 3.1 (on the continuity of the Kadison decomposition). Let be $\Omega$ a topological space, $A, B C^{*}$-algebras, and $\Phi_{t}: A \rightarrow B, t \in \Omega$, surjective linear isometries such that $\Omega \ni t \mapsto \Phi_{t}(a)$ is norm continuous for every $a \in A$. Let us denote by $\Phi_{t}=u_{t} \pi_{t}$ the Kadison decomposition of $\Phi_{t}, t \in \Omega$. Then

$$
\left\{\begin{array}{l}
\Omega \ni t \mapsto \pi_{t}(a) \text { is norm continuous for every } a \in A \\
\Omega \ni t \mapsto \pi_{t}^{-1}(b) \text { is norm continuous for every } b \in B ;
\end{array}\right.
$$

Proof. The first statement in (1) follows by noticing that, for every $0 \leq a \in$ A,

$$
\pi_{t}(a)=\pi_{t}\left(a^{1 / 2}\right)^{2}=\pi_{t}\left(a^{1 / 2}\right) u_{t}^{*} u_{t} \pi_{t}\left(a^{1 / 2}\right)=\Phi_{t}\left(a^{1 / 2}\right)^{*} \Phi_{t}\left(a^{1 / 2}\right) .
$$

The second statement is implied by the first one: For every $b \in B$,

$$
\left\|\pi_{t}^{-1}(b)-\pi_{s}^{-1}(b)\right\|=\left\|\pi_{s}\left(\pi_{t}^{-1}(b)\right)-b\right\| \stackrel{s \rightarrow t}{\rightarrow}\left\|\pi_{t}\left(\pi_{t}^{-1}(b)\right)-b\right\|=0 .
$$

For (2) let $x^{*}=x \in M(A), t_{\iota} \rightarrow t$ in $\Omega, b^{*}=b \in B$ and $\varepsilon>0$ be arbitrary. Let us also consider an (increasing, positive) approximate unit $\left(a_{k}\right)_{k}$ for $A$. Then, for some $k$,

$$
\left\|\left(1_{A^{* *}}-a_{k}\right) x \pi_{t}^{-1}(b)\right\|\left\|\pi_{t}^{-1}(b)\left(1_{A^{* *}}-a_{k}\right)\right\| \leq \frac{\varepsilon}{8\|x\|} .
$$

By the just proved (1) there exists $\iota_{\varepsilon}$ such that, for every $\iota \geq \iota_{\varepsilon}$,

$$
\left\|\pi_{t_{\iota}}^{-1}(b)-\pi_{t}^{-1}(b)\right\| \leq \frac{\varepsilon}{8\|x\|}, \quad\left\|\pi_{t_{\iota}}\left(a_{k} x\right)-\pi_{t}\left(a_{k} x\right)\right\| \leq \frac{\varepsilon}{4\|b\|} .
$$

Then we have for every $\iota \geq \iota_{\varepsilon}$

$$
\begin{aligned}
\| & \left(M\left(\pi_{t_{\iota}}\right)(x)-M\left(\pi_{t}\right)(x)\right) b \| \\
\leq & \left\|M\left(\pi_{t_{\iota}}\right)\left(x-a_{k} x\right) b\right\|+\left\|\left(\pi_{t_{\iota}}\left(a_{k} x\right)-\pi_{t}\left(a_{k} x\right)\right) b\right\| \\
& +\left\|M\left(\pi_{t}\right)\left(a_{k} x-x\right) b\right\| \\
= & \left\|M\left(\pi_{t_{\iota}}\right)\left(x-a_{k} x\right) \cdot M\left(\pi_{t_{\iota}}\right)\left(\pi_{t_{\iota}}^{-1}(b)\right)\right\| \\
& +\left\|M\left(\pi_{t}\right)\left(x-a_{k} x\right) \cdot M\left(\pi_{t}\right)\left(\pi_{t}^{-1}(b)\right)\right\| \\
& +\left\|\left(\pi_{t_{\iota}}\left(a_{k} x\right)-\pi_{t}\left(a_{k} x\right)\right) b\right\| \text { by }(3.1) \\
\leq & \left\|\left(x-a_{k} x\right) \pi_{t_{\iota}}^{-1}(b)\right\|+\left\|\pi_{t_{\iota}}^{-1}(b)\left(x-a_{k} x\right)\right\| \\
& +\left\|\left(x-a_{k} x\right) \pi_{t}^{-1}(b)\right\|+\left\|\pi_{t}^{-1}(b)\left(x-a_{k} x\right)\right\| \\
& \left.+\| \pi_{t_{\iota}}\left(a_{k} x\right)-\pi_{t}\left(a_{k} x\right)\right) b \|
\end{aligned}
$$




$$
\begin{aligned}
\leq & \left\|\left(x-a_{k} x\right)\left(\pi_{t_{\iota}}^{-1}(b)-\pi_{t}^{-1}(b)\right)\right\|+\left\|\left(\pi_{t_{\iota}}^{-1}(b)-\pi_{t}^{-1}(b)\right)\left(x-a_{k} x\right)\right\| \\
& +2\left\|\left(1_{A^{* *}}-a_{k}\right) x \pi_{t}^{-1}(b)\right\|+2\left\|\pi_{t}^{-1}(b)\left(1_{A^{* *}}-a_{k}\right) x\right\| \\
& +\left\|\left(\pi_{t_{\iota}}\left(a_{k} x\right)-\pi_{t}\left(a_{k} x\right)\right) b\right\| \text { and } \iota_{\varepsilon} \\
\leq & \|x\| \frac{\varepsilon}{8\|x\|}+\frac{\varepsilon}{8\|x\|}\|x\|+2 \frac{\varepsilon}{8}+2 \frac{\varepsilon}{8\|x\|}\|x\|+\frac{\varepsilon}{4\|b\|}\|b\|=\varepsilon .
\end{aligned}
$$

For (3) let $b \in B$ be arbitrary. Since

$$
\begin{aligned}
& \left\|u_{t} b-u_{s} b\right\| \\
& =\left\|u_{t} \pi_{t}\left(\pi_{t}^{-1}(b)\right)-u_{s} \pi_{s}\left(\pi_{s}^{-1}(b)\right)\right\| \\
& =\left\|\Phi_{t}\left(\pi_{t}^{-1}(b)\right)-\Phi_{s}\left(\pi_{s}^{-1}(b)\right)\right\| \\
& \leq\left\|\Phi_{t}\left(\pi_{t}^{-1}(b)\right)-\Phi_{s}\left(\pi_{t}^{-1}(b)\right)\right\|+\left\|\pi_{t}^{-1}(b)-\pi_{s}^{-1}(b)\right\|,
\end{aligned}
$$

by the continuity assumption on $t \mapsto \Phi_{t}$ and by (1), $\left\|u_{t} b-u_{s} b\right\| \stackrel{s \rightarrow t}{\rightarrow} 0$. Applying the above to $b$ replaced by $u_{t}^{*} b^{*}$, it follows also that

$$
\left\|b u_{t}-b u_{s}\right\|=\left\|u_{t}^{*} b^{*}-u_{s}^{*} b^{*}\right\|=\left\|u_{s} u_{t}^{*} b^{*}-b^{*}\right\|
$$

converges to $\left\|u_{t} u_{t}^{*} b^{*}-b^{*}\right\|=0$ for $s \rightarrow t$.

It follows in a straightforward way the following:

Theorem 3.2 (on extension of groups of linear isometries). Let $A$ be $a$ $C^{*}$-algebra, and $G$ a topological group. For every strongly continuous group $\left(\Phi_{t}\right)_{t \in G}$ of linear isometries on $A$, the group $\left(M\left(\Phi_{t}\right)\right)_{t \in G}$ is strictly continuous. If $G$ is locally compact, then also conversely, every $\sigma\left(M(A), A^{*}\right)$ continuous group $\left(\tilde{\Phi}_{t}\right)_{t \in G}$ of $\sigma\left(M(A), A^{*}\right)$-continuous (automatic, if $A$ separable or simple!) linear isometries on $M(A)$ leaves $A$ invariant and induces on $A$ a strongly continuous group $(\tilde{\Phi} \mid A)_{t \in G}$ of linear isometries. In particular, $\left(\tilde{\Phi}_{t}=M(\tilde{\Phi} \mid A)\right)_{t \in G}$ is strictly continuous.

Proof. Denoting by $\Phi_{t}=u_{t} \pi_{t}$ the Kadison decomposition of $\Phi_{t}$, the above lemma yields the strict continuity of

$$
G \ni t \mapsto M\left(\Phi_{t}\right)(x)=u_{t} \cdot M\left(\pi_{t}\right)(x)
$$

for all $x \in M(A)$.

The converse statement follows from the theorem in the preceeding section and from the well known fact, according to which all $\sigma\left(A, A^{*}\right)$-continuous locally compact groups of bounded linear operators on $A$ are strongly continuous.

For $\left(\Phi_{t}\right)_{t \in G}$ as above it is convenient to denote the extension $\left(M\left(\Phi_{t}\right)\right)_{t \in G}$ also by $\left(M(\Phi)_{t}\right)_{t \in G}$, underlining in this way that not only the individual $\Phi_{t}$ 's are extended by strict continuity, but the whole strongly continuous group $\Phi$ is extended to a strictly continuous group $M(\Phi)$. 
Next we look for connection between the analytic generator $\Phi_{-i}$ of a strongly continuous one-parameter group $\left(\Phi_{t}\right)_{t \in \mathbb{R}}$ of linear isometries on a $C^{*}$-algebra and the analytic generator $M(\Phi)_{-i}$ of the strictly continuous one-parameter group $\left(M(\Phi)_{t}\right)_{t \in \mathbb{R}}$ (compare with [Kus], Th. 2.41):

Theorem 3.3 (on extension of analytic generators). Let $A$ be a $C^{*}$-algebra, $\left(\Phi_{t}\right)_{t \in \mathbb{R}}$ a strongly continuous one-parameter group of linear isometries on $A$, and $z \in \mathbb{C}$. Then the graph of $M(\Phi)_{z}$ is the closure of the graph of $\Phi_{z}$ with respect to the product of the strict topologies on $M(A) \times M(A)$.

Proof. First of all, the graph of $M(\Phi)_{z}$ is closed with respect to the product of the strict topologies on $M(A) \times M(A)$ according to the corollary in the first section. Since the strict dual of $M(A)$ is $A^{*}$, it is enough to show that the graph of $\Phi_{z}$ is $\sigma\left(M(A), A^{*}\right) \times \sigma\left(M(A), A^{*}\right)$-dense in the graph of $M(\Phi)_{z}$.

By Theorem 1.1 both $\sigma\left(M(A), A^{*}\right)$ and $\sigma\left(A^{*}, M(A)\right)$ have the Krein property, so the formula

$$
\begin{gathered}
\left\langle T_{n}(x), \varphi\right\rangle=\sqrt{\frac{n}{\pi}} \int_{-\infty}^{+\infty} e^{-n t^{2}}\left\langle M(\Phi)_{t}(x), \varphi\right\rangle d t \\
\left\langle T_{n, z}(x), \varphi\right\rangle=\sqrt{\frac{n}{\pi}} \int_{-\infty}^{+\infty} e^{-n(t-z)^{2}}\left\langle M(\Phi)_{t}(x), \varphi\right\rangle d t, x \in M(A), \varphi \in A^{*}
\end{gathered}
$$

define $\sigma\left(M(A), A^{*}\right)$-continuous linear maps

$$
T_{n}, T_{n, z}: M(A) \rightarrow M(A)
$$

(see e.g., [C-Z], Prop. 1.4). In particular,

$$
\begin{aligned}
\Gamma_{n}=\left\{\left(T_{n}(a), T_{n, z}(a)\right) ; a \in A\right\} & \text { is } \sigma\left(M(A), A^{*}\right) \times \sigma\left(M(A), A^{*}\right) \text {-dense } \\
& \text { in }\left\{\left(T_{n}(x), T_{n, z}(x)\right) ; x \in M(A)\right\} .
\end{aligned}
$$

According to $[\mathbf{C}-\mathbf{Z}]$, Cor 2.5 and the proof of Lemma 2.2,

$$
\begin{aligned}
& T_{n} M(\Phi)_{z} \subset M(\Phi)_{z} T_{n}=T_{n, z}, \quad n \geq 1, \\
& \sigma\left(M(A), A^{*}\right)-\lim _{n \rightarrow \infty} T_{n}(x)=x, \quad x \in M(A) .
\end{aligned}
$$

It follows that

$$
\begin{aligned}
& \bigcup_{n \geq 1}\left\{\left(T_{n}(x), T_{n, z}(x)\right) ; x \in M(A)\right\}=\left\{\left(T_{n}(x), M(\Phi)_{z} T_{n}(x)\right) ; x \in M(A)\right\} \\
\supset & \bigcup_{n \geq 1}\left\{\left(T_{n}(x), T_{n} M(\Phi)_{z}(x)\right) ; x \in \mathcal{D}_{M(\Phi)_{z}}\right\},
\end{aligned}
$$

hence also

$$
\bigcup_{n \geq 1} \Gamma_{n}
$$

is $\sigma\left(M(A), A^{*}\right) \times \sigma\left(M(A), A^{*}\right)$-dense in the graph of $M(\Phi)_{z}$. 
But $\bigcup_{n \geq 1} \Gamma_{n}$ is contained in the graph of $\Phi_{z}$. Indeed, since also $\sigma\left(A, A^{*}\right)$ and $\sigma\left(A^{*}, A\right)$ have the Krein property, applying the above quoted results from $[\mathbf{C}-\mathbf{Z}]$ to $\left(\Phi_{t}\right)_{t \in \mathbb{R}}$ instead of $\left(M(\Phi)_{t}\right)_{t \in \mathbb{R}}$, we get

$$
T_{n} A \subset A, T_{n, z} A \subset A \text { and } \Phi_{z} T_{n}\left|A=T_{n, z}\right| A, \quad n \geq 1 .
$$

Therefore

$\left(T_{n}(a), T_{n, z}(a)\right)=\left(T_{n}(a), \Phi_{z} T_{n}(a)\right) \in$ graph of $\Phi_{z}$ for all $n \geq 1$ and $a \in A$.

Now let $A, B$ be $C^{*}$-algebras, and $\pi: A \rightarrow B$ a Jordan $*$-isomorphism. According to the properties of Jordan $*$-homomorphisms, listed at the beginning of the second section,

$$
\pi^{* *}(x z)=\pi^{* *}(x) \pi^{* *}(z)
$$

for every $x \in A^{* *}$ and $z$ in the centre $Z\left(A^{* *}\right)$ of $A^{* *}$. Thus, denoting by $p_{0}(A)$ the greatest projection in $Z\left(A^{* *}\right)$ with $p_{0}(A) A^{* *} \subset Z\left(A^{* *}\right)$, and by $p_{0}(B)$ the similar projection in $Z\left(B^{* *}\right)$, we have

$$
\pi^{* *}\left(p_{0}(A)\right)=p_{0}(B) .
$$

By a well known result due to N. Jacobson, C. Rickart and R.V. Kadison $([\mathbf{K a d} 1]$, Th. 10, see also $[\mathbf{K a d} 2],[\mathbf{S t m}],[\mathbf{T h}])$, there exists a unique projection $p=p(\pi) \in Z\left(B^{* *}\right)$ such that

$$
\left\{\begin{array}{l}
p \leq 1_{B^{* *}}-p_{0}(B), \\
\left(\pi\left(a_{1} a_{2}\right)-\pi\left(a_{1}\right) \pi\left(a_{2}\right)\right) p=0, \quad a_{1}, a_{2} \in A, \\
\left(\pi\left(a_{1} a_{2}\right)-\pi\left(a_{2}\right) \pi\left(a_{1}\right)\right)\left(1_{B^{* *}}-p_{0}(B)-p\right)=0, \quad a_{1}, a_{2} \in A .
\end{array}\right.
$$

We call $p(\pi)$ the strict homomorphic carrier of $\pi$ (compare with [Kad2], Remark (2.7), where the maximal homomorphic carrier $p_{0}(B)+p(\pi)$ is considered).

We notice that actually $p(\pi)$ is the unique element $0 \leq p \in Z\left(B^{* *}\right)$ satisfying (3.2). Indeed, for any such element $p$, denoting by $p_{1}$ and $p_{2}$ the supports of $p$ respectively $1_{B^{* *}}-p_{0}(B)-p$, we have for all $a_{1}, a_{2} \in A$

$$
\begin{aligned}
& \left(\pi\left(a_{1} a_{2}\right)-\pi\left(a_{1}\right) \pi\left(a_{2}\right)\right) p_{1}=0, \\
& \left(\pi\left(a_{1} a_{2}\right)-\pi\left(a_{2}\right) \pi\left(a_{1}\right)\right) p_{2}=0,
\end{aligned}
$$

hence

$$
\left.\left(\pi\left(a_{1}\right) \pi\left(a_{2}\right)\right)-\pi\left(a_{2}\right) \pi\left(a_{1}\right)\right) p_{1} p_{2}=0
$$


It follows successively

$$
\begin{gathered}
p_{1} p_{2} \leq p_{0}(B) \\
p_{1} p_{2}=p_{1} p_{2}\left(1_{B^{* *}}-p_{0}(B)\right)=0 \\
p_{1}-p=p_{1}\left(1_{B^{* *}}-p_{0}(B)-p\right)=p_{1} p_{2}\left(1_{B^{* *}}-p_{0}(B)-p\right)=0, \\
p=p_{1} \text { is a projection. }
\end{gathered}
$$

The next result is covered by [Kad2], Lemmas (4.8) and (4.10), but we give a proof for the convenience of the reader:

Lemma 3.4 (on the continuous dependence of the homomorphic carrier). Let be $\Omega$ a topological space, $A, B C^{*}$-algebras, and $\pi_{t}: A \rightarrow B, t \in \Omega$, Jordan $*$-isomorphisms such that

$$
\Omega \ni t \mapsto \pi_{t}(a) \in B^{* *} \text { is weak }{ }^{*} \text {-continuous for every } a \in A \text {. }
$$

Then

$$
\begin{gathered}
\Omega \ni t \mapsto \pi_{t}(a) \in B^{* *} \text { is } s^{*} \text {-continuous for every } a \in A, \\
\Omega \ni t \mapsto p\left(\pi_{t}\right) \in Z\left(B^{* *}\right) \text { is s-continuous. }
\end{gathered}
$$

Proof. Clearly, it is enough to prove the s $^{*}$-continuity of $\Omega \ni t \mapsto \pi_{t}(a) \in$ $B^{* *}$ for all self-adjoint $a \in A$. But in this case

$$
\left(\pi_{t}(a)-\pi_{s}(a)\right)^{2}=\pi_{t}\left(a^{2}\right)+\pi_{s}\left(a^{2}\right)-\pi_{t}(a) \pi_{s}(a)-\pi_{s}(a) \pi_{t}(a)
$$

is weak*-convergent to

$$
2 \pi_{t}\left(a^{2}\right)-2 \pi_{t}(a) \pi_{t}(a)=0
$$

for $s \rightarrow t$.

Let be $t_{\iota} \rightarrow t$ in $\Omega$. If $p$ is any weak*-limit point of $\left(p\left(\pi_{t_{\iota}}\right)\right)_{\iota}$ then

$$
\begin{aligned}
& p\left(\pi_{t_{\iota}}\right) \leq 1_{B^{* *}}-p_{0}(B), \\
& \left(\pi_{t_{\iota}}\left(a_{1} a_{2}\right)-\pi_{t_{\iota}}\left(a_{1}\right) \pi_{t_{\iota}}\left(a_{2}\right)\right) p\left(\pi_{t_{\iota}}\right)=0 \\
& \left(\pi_{t_{\iota}}\left(a_{1} a_{2}\right)-\pi_{t_{\iota}}\left(a_{2}\right) \pi_{t_{\iota}}\left(a_{1}\right)\right)\left(1_{B^{* *}}-p_{0}(B)-p\left(\pi_{t_{\iota}}\right)\right)=0,
\end{aligned}
$$

true for all $\iota$ and $a_{1}, a_{2} \in A$, together with

$$
\pi_{t_{\iota}}(a) \stackrel{s^{*}}{\rightarrow} \pi_{t}(a), \quad a \in A,
$$

implies

$$
\begin{aligned}
& p \leq 1_{B^{* *}}-p_{0}(B), \\
& \left(\pi_{t}\left(a_{1} a_{2}\right)-\pi_{t}\left(a_{1}\right) \pi_{t}\left(a_{2}\right)\right) p=0 \\
& \left(\pi_{t}\left(a_{1} a_{2}\right)-\pi_{t}\left(a_{2}\right) \pi_{t}\left(a_{1}\right)\right)\left(1_{B^{* *}}-p_{0}(B)-p\right)=0
\end{aligned}
$$

for all $a_{1}, a_{2} \in A$. By the remark before the statement of the lemma it follows that $p=p\left(\pi_{t}\right)$. Thus $p\left(\pi_{t_{\iota}}\right) \rightarrow p\left(\pi_{t}\right)$ in the weak* topology.

But on the projections of $B^{* *}$ the weak ${ }^{*}$ topology coincides with the stopology: For $e, f \in B^{* *}$ projections

$$
(e-f)^{2}=e-f-f(e-f)-(e-f) f \stackrel{\text { weak }^{*}}{\longrightarrow} 0
$$


whenever $e \stackrel{\text { weak }^{*}}{\longrightarrow} f$. Consequently

$$
p\left(\pi_{t_{\iota}}\right) \rightarrow p\left(\pi_{t}\right) \text { in the } s \text {-topology. }
$$

The above lemmas on the continuity of the Kadison decomposition respectively on the continuous dependence of the homomorphic carrier allow us to prove the following:

Theorem 3.5 (on the structure of strongly continuous connected groups of linear isometries). Let $A$ be a $C^{*}$-algebra, $G$ a connected topological group, and $\left(\Phi_{t}\right)_{t \in G}$ a strongly continuous group of linear isometries on $A$. Let

$$
\Phi_{t}=u_{t} \pi_{t}, \quad t \in G
$$

denote the corresponding Kadison decompositions. Then $\left(\pi_{t}\right)_{t \in G}$ is a strongly continuous group of $*$-automorphisms of $A$, while $G \ni t \mapsto u_{t} \in M(A)$ is a strictly continuous unitary $M(\pi)$-cocycle:

$$
u_{t s}=u_{t} \cdot M\left(\pi_{t}\right)\left(u_{s}\right), \quad t, s \in G .
$$

Proof. We recall that the strict continuity of $G \ni t \mapsto u_{t}$ is exactly statement (3) in Lemma 3.1. Also the $M(\pi)$-cocycle property follows easily:

$$
u_{t s}=M\left(\Phi_{t s}\right)\left(1_{A^{* *}}\right)=M\left(\Phi_{t}\right)\left(M\left(\Phi_{s}\right)\left(1_{A^{* *}}\right)\right)=u_{t} M\left(\pi_{t}\right)\left(u_{s}\right) .
$$

Using it, we get successively for every $a \in A$

$$
\begin{gathered}
u_{t s} \pi_{t s}(a)=\Phi_{t s}(a)=\Phi_{t}\left(\Phi_{s}(a)\right)=u_{t} \pi_{t}\left(u_{s} \pi_{s}(a)\right), \\
\pi_{t s}(a)=u_{t s}^{*} u_{t} \pi_{t}\left(u_{s} \pi_{s}(a)\right)=M\left(\pi_{t}\right)\left(u_{s}^{*}\right) \cdot \pi_{t}\left(u_{s} \pi_{s}(a)\right) .
\end{gathered}
$$

Consequently,

$$
\pi_{t} \text { multiplicative } \Rightarrow \pi_{t s}=\pi_{t} \cdot \pi_{s} \text { for all } s \in G .
$$

According to this and to statement (1) in Lemma 3.1, it remains only to prove the multiplicativity of every $\pi_{t}$.

For we shall use the arguments from the proof of [Kad2], Th. 3.4:

Let $e$ denote the neutral element of $G$. By the two continuity lemmas in this section, the map

$$
G \ni t \mapsto p_{t}=p_{0}(A)+p\left(\pi_{t}\right) \in Z\left(A^{* *}\right)
$$

is $s$-continuous. Since $\pi_{e}=i d_{A}$ is multiplicative, we have $p_{e}=1_{A^{* *}}$.

Now every pure state $\varphi$ of $A$ is multiplicative on $Z\left(A^{* *}\right)$, so

$$
\langle p, \varphi\rangle=1 \text { or } 0 \text { for all projections } p \in Z\left(A^{* *}\right) .
$$

$\mathrm{G}$ being connected, the continuous function

$$
G \ni t \mapsto\left\langle p_{t}, \varphi\right\rangle \in\{1.0\}
$$


is constant, therefore

$$
\left\langle p_{t}, \varphi\right\rangle=\left\langle p_{e}, \varphi\right\rangle=1 \text { for every } t \in G .
$$

We conclude that, for every $t \in G$, every $a_{1}, a_{2} \in A$ and every pure state $\varphi$ of $A$,

$$
\begin{aligned}
& \left\langle\left|\pi_{t}\left(a_{1} a_{2}\right)-\pi_{t}\left(a_{1}\right) \pi_{t}\left(a_{2}\right)\right|^{2}, \varphi\right\rangle \\
& =\left\langle\left(1_{A^{* *}}-p_{t}\right)\left|\pi_{t}\left(a_{1} a_{2}\right)-\pi_{t}\left(a_{1}\right) \pi_{t}\left(a_{2}\right)\right|^{2}\left(1_{A^{* *}}-p_{t}\right), \varphi\right\rangle \\
& \leq 4\left\|a_{1}\right\|^{2}\left\|a_{2}\right\|^{2}\left\langle\left(1_{A^{* *}}-p_{t}\right), \varphi\right\rangle=0,
\end{aligned}
$$

hence

$$
\pi_{t}\left(a_{1} a_{2}\right)-\pi_{t}\left(a_{1}\right) \pi_{t}\left(a_{2}\right)=0
$$

Let $A$ be a $C^{*}$-algebra, and $G$ a topological group. A strongly continuous group $\left(\Phi_{t}\right)_{t \in G}$ of linear isometries on $A$ is called in [Kus], Section 2 semimultiplicative whenever there are strongly continuous groups $\left(\Phi_{t}^{(j)}\right)_{t \in G}, j=$ 1,2 , of linear isometries on $A$ such that

$$
\Phi_{t}(a b)=\Phi_{t}(a) \Phi_{t}^{(1)}(b)=\Phi_{t}^{(2)}(a) \Phi_{t}(b), t \in G, a, b \in A .
$$

But it is easy to see that, with $\Phi_{t}=u_{t} \pi_{t}$ the Kadison decomposition of $\Phi_{t}$, for any maps $\Phi_{t}^{(j)}: A \rightarrow A, t \in G, j=1,2$, satisfying (3.3), we have necessarily

$$
\Phi_{t}^{(1)}=\pi_{t} \text { and } \Phi_{t}^{(2)}=u_{t} \pi_{t} u_{t}^{*}, \quad t \in G .
$$

Thus $\left(\Phi_{t}\right)_{t \in G}$ is semi-multiplicative if and only if all $\pi_{t}$ 's are $*$-automorphisms and the above theorem claims essentially that, assuming $G$ connected, every strongly continuous representation of $G$ by linear isometries on $A$ is automatically semi-multiplicative.

We notice that our Theorem 3.3 on the extension of analytic generators was proved in [Kus], Th. 2.41 under the additional assumption of semimultiplicativity of the one-parameter group, which turns out to be automatic by the above remark. However, our approach is more natural for the prevailingly linear character of the statement of the theorem. Indeed, our proof works for any (even not bounded) strongly continuous one-parameter group of bounded linear operators on $A$, which extends by strict continuity to a $\sigma\left(A^{*}, M(A)\right)$-continuous one-parameter group of bounded linear operators on $M(A)$.

Let us consider also the $W^{*}$-algebra counterpart of the above theorem (compare with [Kad2], Th. 3.4):

Theorem 3.6 (on the structure of weak* continuous connected groups of linear isometries). Let $M$ be a $W^{*}$-algebra, whose centre is atomic, $G$ a 
connected topological group, and $\left(\Phi_{t}\right)_{t \in G}$ a weak ${ }^{*}$ continuous group of linear isometries on $M$. Let

$$
\Phi_{t}=u_{t} \pi_{t}, \quad t \in G
$$

denote the corresponding Kadison decompositions. Then $\left(\pi_{t}\right)_{t \in G}$ is an $s^{*}$ continuous group of $*$-automorphisms of $M$, while $G \ni t \mapsto u_{t} \in M$ is an $s^{*}$-continuous unitary $\pi$-cocycle:

$$
u_{t s}=u_{t} \pi_{t}\left(u_{s}\right), \quad t, s \in G .
$$

Proof. First of all, $G \ni t \mapsto u_{t}=\Phi_{t}\left(1_{M}\right)$ is weak*-continuous, hence also $s^{*}$-continuous. Indeed, on the unitaries of $M$ the weak* topology coincides with the $s^{*}$-topology: For $u, v \in M$ unitaries

$$
(u-v)^{*}(u-v)+(u-v)(u-v)^{*}=4 \cdot 1_{M}-u^{*} v-v^{*} u-u v^{*}-v u^{*} \stackrel{\text { weak }^{*}}{\longrightarrow} 0
$$

whenever $v \stackrel{\text { weak }^{*}}{\longrightarrow} u$.

Next, for every $x \in M$,

$$
G \ni t \mapsto \pi_{t}(x)=u_{t}^{*} \Phi_{t}(x) \in M
$$

is weak*-continuous according to the weak*-continuity of $\left(\Phi_{t}\right)_{t \in G}$ and the $s$-continuity of $G \ni t \mapsto u_{t}$. It follows by straightforward arguments that the maps

$$
G \ni t \mapsto \pi_{t}(x) \in M, \quad x \in M
$$

are even $s^{*}$-continuous (see $[\mathbf{K a d} 2]$, Lemma 4.10 or the first paragraph of the proof of Lemma 3.4).

Similarly, as in the proof of the preceeding theorem, we get also

$$
u_{t s}=u_{t} \pi_{t}\left(u_{s}\right), \quad t, s \in G,
$$

and then

$$
\pi_{t} \text { multiplicative } \Rightarrow \pi_{t s}=\pi_{t} \cdot \pi_{s} \text { for all } s \in G .
$$

Thus it remains only to prove the multiplicativity of every $\pi_{t}$.

Let $p_{0}$ denote the greatest projection in the centre $Z(M)$ of $M$ satisfying $p_{0} M \subset Z(M)$. Then

$$
\pi_{t}\left(p_{0}\right)=p_{0} \quad \text { for all } t \in G .
$$

According to $[\operatorname{Kad} 1]$, Th. 10, for every $t \in G$ there exists a unique projection $p\left(\pi_{t}\right) \in Z(M)$ such that

$$
\begin{aligned}
& p\left(\pi_{t}\right) \leq 1_{M}-p_{0}, \\
& \left(\pi_{t}\left(x_{1} x_{2}\right)-\pi_{t}\left(x_{1}\right) \pi_{t}\left(x_{2}\right)\right) p\left(\pi_{t}\right)=0, \quad x_{1}, x_{2} \in M, \\
& \left(\pi_{t}\left(x_{1} x_{2}\right)-\pi_{t}\left(x_{2}\right) \pi_{t}\left(x_{1}\right)\right)\left(1_{M}-p_{0}-p\left(\pi_{t}\right)\right)=0, \quad x_{1}, x_{2} \in M .
\end{aligned}
$$


Moreover, arguing similarly as in the proof of Lemma 3.4, it is easy to verify that the map

$$
G \ni t \mapsto p\left(\pi_{t}\right) \in Z(M)
$$

hence also

$$
G \ni t \mapsto p_{t}=p_{0}+p\left(\pi_{t}\right) \in Z(M)
$$

is $s$-continuous. We notice that $\pi_{e}=i d_{M}$ implies $p_{e}=1_{M}$, where $e$ stands for the neutral element of $G$.

Now, for every normal state $\varphi$ on $M$, whose central support is a minimal projection of $Z(M)$, the continuous function

$$
G \ni t \mapsto\left\langle p_{t}, \varphi\right\rangle
$$

takes values in $\{1,0\}$. $G$ being connected, it follows that

$$
\left\langle p_{t}, \varphi\right\rangle=\left\langle p_{e}, \varphi\right\rangle=1 \text { for all } t \in G .
$$

Consequently, for every $t \in G$ and $x_{1}, x_{2} \in M$,

$$
\begin{aligned}
& \left\langle\left|\pi_{t}\left(x_{1} x_{2}\right)-\pi_{t}\left(x_{1}\right) \pi_{t}\left(x_{2}\right)\right|^{2}, \varphi\right\rangle \\
& =\left\langle\left(1_{M}-p_{t}\right)\left|\pi_{t}\left(x_{1} x_{2}\right)-\pi_{t}\left(x_{1}\right) \pi_{t}\left(x_{2}\right)\right|^{2}\left(1_{M}-p_{t}\right), \varphi\right\rangle \\
& \leq 4\left\|x_{1}\right\|^{2}\left\|x_{2}\right\|^{2}\left\langle 1_{M}-p_{t}, \varphi\right\rangle=0 .
\end{aligned}
$$

Since $Z(M)$ is atomic, the normal states on $M$ with central supports minimal in $Z(M)$ separate the points of $M$ and we conclude that

$$
\pi_{t}\left(x_{1} x_{2}\right)-\pi_{t}\left(x_{1}\right) \pi_{t}\left(x_{2}\right)=0 \text { for all } t \in G \text { and } x_{1}, x_{2} \in M .
$$

In particular, if $M$ is a factor, $G$ is a connected topological group and $\left(\Phi_{t}\right)_{t \in G}$ is a weak*-continuous group of linear isometries on $M$, then

$$
\Phi_{t}=u_{t} \pi_{t}, \quad t \in G
$$

with $\left(\pi_{t}\right)_{t \in G}$ an $s^{*}$-continuous group of $*$-automorphisms of $M$ and $G \ni t \mapsto$ $u_{t} \in M$ an $s^{*}$-continuous unitary $\pi$-cocycle.

However, the above theorem does not hold without the assumption on the centre of $M$, even we assume $M$ of type $I_{2}, G=\mathbb{R}$ and all $\Phi_{t}$ Jordan *-automorphisms. Indeed, denoting by $M$ the $W^{*}$-algebra $L^{\infty}\left(\mathbb{R} ; \operatorname{Mat}_{2}(\mathbb{C})\right)$ of all essentially bounded (equivalence classes of) $2 \times 2$ matrix valued measurable functions on $\mathbb{R}$, choosing some $*$-anti-automorphism $\sigma$ of $\mathrm{Mat}_{2}(\mathbb{C})$ (e.g., the transpose map) and putting for $F \in L^{\infty}\left(\mathbb{R} ; \operatorname{Mat}_{2}(\mathbb{C})\right.$ )

$$
\begin{aligned}
& \Phi_{t}(F)(s)=\left\{\begin{array}{l}
\sigma(F(s-t)) \text { if } 0<s<t \\
F(s-t) \text { otherwise, }
\end{array} \quad t \geq 0, s \in \mathbb{R},\right. \\
& \Phi_{t}(F)(s)=\left\{\begin{array}{l}
\sigma^{-1}(F(s-t)) \text { if } t<s<0 \\
F(s-t) \text { otherwise, }
\end{array} \quad t \leq 0, s \in \mathbb{R},\right.
\end{aligned}
$$


$\left(\Phi_{t}\right)_{t \in \mathbb{R}}$ is a weak ${ }^{*}$-continuous one-parameter group of Jordan $*$-automorphisms of $M$, but no $\Phi_{t}$ with $t \neq 0$ is multiplicative.

We finish the section (and the paper) with a continuity criterion for groups of linear isometries on multiplier algebras of separable $C^{*}$-algebras and with a result concerning continuity on corona algebras of separable $C^{*}$-algebras.

They will follow from the next two lemmas of general character:

Lemma $3.7([\mathbf{Z 2}])$. Let $(X, \mathcal{F})$ be a dual pair of Banach spaces, $G$ a locally compact group, and $\left(U_{t}\right)_{t \in G}$ a group of $\sigma(X, \mathcal{F})$-continuous linear operators on $X$. If there exists a compact set $K \subset G$ of nonzero Haar measure, such that all functions

$$
K \ni t \mapsto\left\langle U_{t}(x), \varphi\right\rangle, \quad x \in X, \varphi \in \mathcal{F}
$$

are continuous, then $\left(U_{t}\right)_{t \in G}$ is $\sigma(X, \mathcal{F})$-continuous.

Proof. Let $m$ denote a left Haar measure on $G$. By [He-R], Cor. 20.17 there exists $t_{0} \in G$ such that $m\left(K \cap t_{0} K^{-1}\right)>0$. Then the support of the restriction of $m$ to $K \cap t_{0} K^{-1}$ is a compact subset $K_{0}$ of $K \cap t_{0} K^{-1}$ such that

$$
\begin{gathered}
m\left(K_{0}\right)=m\left(K \cap t_{0} K^{-1}\right)>0, \text { in particular, } K_{0} \neq \emptyset, \\
V \subset G \text { open, } V \cap K_{0} \neq \emptyset \Rightarrow m\left(V \cap K_{0}\right)>0 .
\end{gathered}
$$

Now let $x \in X, \varphi \in \mathcal{F}$ and $\varepsilon>0$ be arbitrary. Defining $F: K_{0} \times K_{0} \rightarrow \mathbb{C}$ by

$$
F(t, s)=\left\langle U_{t s^{-1}}(x), \varphi\right\rangle \quad t, s \in K_{0},
$$

$F$ is separately continuous. Indeed, by the continuity assumption on $U$,

$$
K_{0} \subset K \ni t \mapsto\left\langle U_{t s^{-1}}(x), \varphi\right\rangle=\left\langle U_{t}\left(U_{s^{-1}}(x)\right), \varphi\right\rangle
$$

is continuous for every $s \in G$, and

$$
K_{0} \subset t_{0} K^{-1} \ni s \mapsto\left\langle U_{t s^{-1}}(x), \varphi\right\rangle=\left\langle U_{s^{-1} t_{0}}\left(U_{t_{0}^{-1}}(x)\right), U_{t}^{\mathcal{F}}(\varphi)\right\rangle
$$

is continuous for every $t \in G$. According to a theorem of I. Namioka ([N], Th. 1.2, see also $[\mathbf{C h r}]$, Th. 1 ) it follows the existence of a dense $G_{\delta}$ set $D$ in $K_{0}$ such that $F$ is jointly continuous in every point of $D \times K_{0}$. In particular, choosing some $t_{1} \in D, F$ is continuous in $\left(t_{1}, t_{1}\right)$, so there exists some open set $t_{1} \in V_{1} \subset G$ such that

$$
(t, s) \in\left(V_{1} \cap K_{0}\right) \times\left(V_{1} \cap K_{0}\right) \Rightarrow\left|F(t, s)-F\left(t_{1}, t_{1}\right)\right|<\varepsilon,
$$

that is

$$
t \in\left(V_{1} \cap K_{0}\right) \cdot\left(V_{1} \cap K_{0}\right)^{-1} \Rightarrow\left|\left\langle U_{t}(x)-x, \varphi\right\rangle\right|<\varepsilon .
$$

But $V_{1} \cap K_{0} \ni t$, being not empty, we have $m\left(V_{1} \cap K_{0}\right)>0$ and [He-R], Cor. 20.17 implies that $\left(V_{1} \cap K_{0}\right) \cdot\left(V_{1} \cap K_{0}\right)^{-1}$ is a neighborhood of the neutral element of $G$.

We conclude that the mappings

$$
G \ni t \rightarrow U_{t}(x) \in X, \quad x \in X
$$


are $\sigma(X, \mathcal{F})$-continuous in the neutral element of $G$, hence everywhere.

Lemma 3.8 ([Z2], compare with $[\mathbf{H i}-\mathbf{P}]$, Th. 3.5.3 and Th. 10.2.3). Let $X$ be a separable Banach space, $\mathcal{F}$ a linear subspace of the dual $X^{*}$, satisfying

$$
\|x\|=\sup \{|\langle x, \varphi\rangle| ; \varphi \in \mathcal{F},\|\varphi\| \leq 1\}, \quad x \in X,
$$

$G$ a locally compact group with left Haar measure $m$, and $\left(U_{t}\right)_{t \in G}$ a group of bounded linear operators on $X$. If there exists a Haar-measurable $B \subset G$ with $0<m(B)<+\infty$, such that all functions

$$
B \ni t \mapsto\left\langle U_{t}(x), \varphi\right\rangle, \quad x \in X, \varphi \in \mathcal{F}
$$

are Haar-measurable, then $\left(U_{t}\right)_{t \in G}$ is strongly continuous.

Proof. Let $\left(x_{k}\right)_{k \geq 1}$ be a norm dense sequence in $X \backslash\{0\}$. For every $k, n \geq 1$ there exists $\varphi_{k, n} \in \mathcal{F},\left\|\varphi_{k, n}\right\| \leq 1$, such that

$$
\left|\left\langle x_{k}, \varphi_{k, n}\right\rangle\right|>\left\|x_{k}\right\|-\frac{1}{n}
$$

Then

$$
\|x\|=\sup \left\{\left|\left\langle x, \varphi_{k, n}\right\rangle\right| ; k, n \geq 1\right\}, \quad x \in X .
$$

Let $x \in X$ and $\delta, \varepsilon>0$ be arbitrary.

By the measurability assumption on $U$, the functions

$$
B \ni t \mapsto\left\|U_{t}(x)-x_{k}\right\|=\sup \left\{\left|\left\langle U_{t}(x)-x_{k}, \varphi_{p, q}\right\rangle\right| ; p, q \geq 1\right\}, k \geq 1
$$

are Haar measurable, so all sets

$$
S_{k}=\left\{t \in B ;\left\|U_{t}(x)-x_{k}\right\|<\varepsilon\right\} \subset B, \quad k \geq 1
$$

are also Haar-measurable. Since $\left(x_{k}\right)_{k \geq 1}$ is norm dense in $X$, we have

$$
\bigcup_{k \geq 1} S_{k}=B
$$

Thus, putting

$$
R_{k}=S_{k} \backslash \bigcup_{1 \leq l<k} S_{l}, \quad k \geq 1,
$$

we get a partition $R_{1}, R_{2}, \ldots$ of $B$ in Haar-measurable sets such that

$$
t \in R_{k} \Rightarrow\left\|U_{t}(x)-x_{k}\right\|<\varepsilon, \quad k \geq 1 .
$$

By the countable additivity of $m$ there exists $k_{0} \geq 1$ with

$$
m\left(B \backslash \bigcup_{k=1}^{k_{0}} R_{k}\right)<\frac{\delta}{3} m(B),
$$


and then, by the regularity of $m$, a compact set $K_{0} \subset \bigcup_{k=1}^{k_{0}} R_{k}$ with

$$
m\left(\bigcup_{k=1}^{k_{0}} R_{k} \backslash K_{0}\right)<\frac{\delta}{3} m(B) .
$$

It follows that

$$
m\left(B \backslash K_{0}\right)<\frac{2 \delta}{3} m(B)
$$

and the Haar-measurable sets

$$
E_{k}=R_{k} \cap K_{0}, \quad 1 \leq k \leq k_{0}
$$

yield a partition of $K_{0}$ such that

$$
t \in E_{k} \Rightarrow\left\|U_{t}(x)-x_{k}\right\|<\varepsilon, \quad 1 \leq k \leq k_{0} .
$$

Let $\chi_{E_{k}}$ denote the characteristic function of $E_{k}$. By the Lusin theorem there are compact subsets $K_{1}, \ldots, K_{k_{0}}$ of $K_{0}$ such that, for every $1 \leq k \leq k_{0}$,

$$
\begin{gathered}
m\left(K_{0} \backslash K_{k}\right)<\frac{\delta}{3 k_{0}} m(B), \\
\chi_{E_{k}} \mid K_{k}: K_{k} \rightarrow[0,1] \text { is continuous. }
\end{gathered}
$$

Then

$$
K_{x, \delta, \varepsilon}=\bigcap_{k=1}^{k_{0}} K_{k} \subset K_{0}
$$

is a compact subset of $B$ with

$$
m\left(B \backslash K_{x, \delta, \varepsilon}\right)<\delta m(B)
$$

and all functions

$$
\chi_{E_{k}} \mid K_{x, \delta, \varepsilon}: K_{x, \delta, \varepsilon} \rightarrow[0,1]
$$

are continuous. Therefore

$$
K_{x, \delta, \varepsilon} \ni t \mapsto F_{x, \delta, \varepsilon}(t)=\sum_{k=1}^{k_{0}} \chi_{E_{k}}(t) x_{k} \in X
$$

is norm continuous. Moreover,

$$
\left\|U_{t}(x)-F_{x, \delta, \varepsilon}(t)\right\|<\varepsilon \text { for all } t \in K_{x, \delta, \varepsilon} .
$$

Summing up the aboves: For every $x \in X$ and every $\delta, \varepsilon>0$ there exists a compact set $K_{x, \delta, \varepsilon} \subset B$ with

$$
m\left(B \backslash K_{x, \delta, \varepsilon}\right)<\delta m(B)
$$

and a norm continuous map $F_{x, \delta, \varepsilon}: K_{x, \delta, \varepsilon} \rightarrow X$ with

$$
\left\|U_{t}(x)-F_{x, \delta, \varepsilon}(t)\right\|<\varepsilon \text { for all } t \in K_{x, \delta, \varepsilon} .
$$


Then, for every $x \in X$ and $\delta>0$,

$$
K_{x, \delta}=\bigcap_{n=1}^{\infty} K_{x, \delta 2^{-n}, n^{-1}} \subset B
$$

is a compact set with

$$
m\left(B \backslash K_{x, \delta}\right)<\delta m(B)
$$

and

$$
K_{x, \delta} \ni t \rightarrow U_{t}(x) \in X
$$

is norm continuous as the uniform limit of the norm continuous maps $F_{x, \delta 2^{-n}, n^{-1}} \mid K_{x, \delta}, n \geq 1$.

Denoting now

$$
L_{1}=\bigcap_{k=1}^{\infty} K_{x_{k}, \frac{1}{3} 2^{-k}},
$$

$L_{1}$ is compact subset of $B$ with

$$
m\left(B \backslash L_{1}\right)<\frac{1}{3} m(B)
$$

and all mappings

$$
L_{1} \ni t \mapsto U_{t}\left(x_{k}\right) \in X, \quad k \geq 1
$$

are norm continuous.

On the other hand, again by the measurability assumption on $U$, the function

$$
B \ni t \mapsto\left\|U_{t}\right\|=\sup \left\{\left\|x_{k}\right\|^{-1} \cdot\left|\left\langle U_{t}\left(x_{k}\right), \varphi_{p, q}\right\rangle\right| ; k, p, q \geq 1\right\}
$$

is Haar-measurable. By the countable additivity of $m$ there exists a sufficiently large $c>0$ with

$$
m\left(\left\{t \in B ;\left\|U_{t}\right\| \leq c\right\}\right)>\frac{2}{3} m(B),
$$

and then, by the regularity of $m$, a compact set

$$
L_{2} \subset\left\{t \in B ;\left\|U_{t}\right\| \leq c\right\} \text { with } m\left(L_{2}\right)>\frac{2}{3} m(B) .
$$

We conclude that $L=L_{1} \cap L_{2}$ is a compact subset of $B$ such that

$$
\begin{aligned}
m(L) & =m\left(L_{2}\right)-m\left(L_{2} \backslash L_{1}\right) \\
& \geq m\left(L_{2}\right)-m\left(B \backslash L_{1}\right) \\
& >\frac{2}{3} m(B)-\frac{1}{3} m(B)>0, \\
\left\|U_{t}\right\| & \leq c \text { for all } t \in L
\end{aligned}
$$

and the mappings

$$
L \ni t \mapsto U_{t}\left(x_{k}\right) \in X, \quad k \geq 1
$$


are norm continuous. Since $\left(x_{k}\right)_{k \geq 1}$ is dense in $X$, it follows the norm continuity of all mappings

$$
L \ni t \mapsto U_{t}(x) \in X, \quad x \in X .
$$

Now Lemma 3.7 yields the $\sigma\left(X, X^{*}\right)$-continuity, hence the strong continuity of $\left(U_{t}\right)_{t \in G \text {. }}$

By the above result, every representation of a locally compact group by bounded linear operators on a separable Banach space, which has a "minimal regularity property", is automatically strongly continuous.

Theorem 3.9 (on characterizing continuity of linear isometry groups on separable $C^{*}$-algebras and their multipliers algebras). Let be $H$ a complex Hilbert space, $A \subset B(H)$ a non-degenerate, separable $C^{*}$-subalgebra, and

$$
M(A)=\{x \in B(H) ; x a, \text { ax } \in \text { A for all } a \in A\} .
$$

Then $\Phi \mapsto \Phi \mid A$ establishes an one-to-one correspondence between the surjective linear isometries $\Phi$ on $M(A)$ and those on $A$.

Let further be $G$ a locally compact group and $\left(\Phi_{t}\right)_{t \in G}$ a group of linear isometries on $M(A)$. If there are

- a linear subspace $\mathcal{F} \subset B(H)^{*}$, satisfying $\|a\|=\sup \{|\langle a, \varphi\rangle| ; \varphi \in$ $\mathcal{F},\|\varphi\| \leq 1\}$ for all $a \in A$,

- a Haar-measurable $B \subset G$ of nonzero, finite Haar measure, such that all functions

$$
B \ni t \mapsto\left\langle\Phi_{t}(a), \varphi\right\rangle, \quad a \in A, \varphi \in \mathcal{F}
$$

are Haar-measurable, then $(\Phi \mid A)_{t \in G}$ is strongly continuous and $\left(\Phi_{t}\right)_{t \in G}$ is strictly continuous, i.e., the maps

$$
G \ni t \mapsto \Phi_{t}(x) a, \quad G \ni t \mapsto a \Phi_{t}(x)
$$

are norm-continuous for all $x \in M(A)$ and $a \in A$.

Proof. The first statement follows from Theorem 2.3, taking into account the canonical identification of the here defined $M(A)$ with the multiplier algebra defined in the first section (see [Ped1], Prop. 3.12.3 or [WO], Prop. 2.2.11).

Now let $\left(\Phi_{t}\right)_{t \in G}$ be as in the theorem. Then Lemma 3.8 implies the strong continuity of $\left(\Phi_{t} \mid A\right)_{t \in G}$, and then the strict continuity of $\left(\Phi_{t}\right)_{t \in G}$ is entailed by Theorem 3.2 .

In general, for $\left(\Phi_{t}\right)_{t \in G}$ strongly continuous group of linear isometries on a separable $C^{*}$-algebra $A$, the group $\left(C\left(\Phi_{t}\right)\right)_{t \in G}$ is not strongly continuous. However we have strong continuity on separable, invariant $C^{*}$-algebras of $C(A)$ : 
Theorem 3.10 (on continuity properties of linear isometry groups on corona algebras of separable $C^{*}$-algebras). Let be $G$ a locally compact group, $\left(\Phi_{t}\right)_{t \in G}$ a strongly continuous group of linear isometries on a separable $C^{*}$ algebra $A$, and $D$ a separable $C^{*}$-subalgebra of $C(A)=M(A) / A$, left invariant by $\left(C\left(\Phi_{t}\right)\right)_{t \in G}$. Then the group $\left(C\left(\Phi_{t}\right) \mid D\right)_{t \in G}$ of linear isometries on $D$ is strongly continuous.

Proof. Let us denote by $\pi$ the quotient map $M(A) \rightarrow C(A)$ and let $\left(x_{n}\right)_{n \geq 1}$ be a sequence in $M(A)$ such that $\left(\pi\left(x_{n}\right)\right)_{n \geq 1}$ is dense in $D$. Then the $C^{*}$ subalgebra $B \subset M(A)$ generated by $A \cup\left\{x_{n} ; n \geq 1\right\}$ is separable and it is easy to see that

$$
B=\pi^{-1}(D) \text {. }
$$

It follows that $B$ is left invariant by $\left(M\left(\Phi_{t}\right)\right)_{t \in G}$. Since $\left(M\left(\Phi_{t}\right) \mid B\right)_{t \in G}$ is $\sigma\left(B, A^{*}\right)$-continuous, according to Lemma 3.8 it is strongly continuous and the strong continuity of $\left(C\left(\Phi_{t}\right) \mid D\right)_{t \in G}$ follows.

Actually we have proved more: If $G,\left(\Phi_{t}\right)_{t \in G}$ and $\pi$ are as above, then

$$
\pi^{-1}\left(\cup\left\{D ; \begin{array}{c}
D \subset C(A) \text { separable } C^{*} \text {-subalgebra } \\
C\left(\Phi_{t}\right) D \subset D \text { for all } t \in G
\end{array}\right\}\right)
$$

is contained in

$$
\left\{x \in M(A) ; G \ni t \mapsto M\left(\Phi_{t}\right)(x) \text { is norm-continuous }\right\} .
$$

Clearly, the above two $C^{*}$-algebras of $M(A)$ are equal whenever $G$ is separable.

\section{References}

[Ar] W. Arveson, On groups of automorphisms of operator algebras, J. Funct. Analysis, 15 (1974), 217-243.

[B] N. Bourbaki, Topological Vector Spaces, Springer-Verlag, 1987.

[Br] L.G. Brown, Determination of $A$ from $M(A)$ and related matters, C.R. Math. Rep. Acad. Sci. Canada, 10 (1988), 273-278.

[Buc] R.C. Buck, Bounded continuous functions on a locally compact space, Michigan Math. J., 5 (1958), 95-104.

[Bus] R.C. Busby, Double centralizers and extensions of $C^{*}$-algebras, Trans. Amer. Math. Soc., 132 (1968), 79-99.

[Chr] J.P.R. Christensen, Joint continuity of separately continuous functions, Proc. Amer. Math. Soc., 82 (1981), 455-461.

[C-Z] I. Ciorănescu and L. Zsidó, Analytic generators for one-parameter groups, Tohoku Math. J., 28 (1976), 327-362.

[Cnw] J.B. Conway, The strict topology and compactness in the space of measures, II, Trans. Amer. Math. Soc., 126 (1967), 474-486.

[D-Z] C. D'Antoni and L. Zsidó, Abelian strict approximation in multiplier $C^{*}$-algebras, preprint, 1998. 
[He-R] E. Hewitt and K.A. Ross, Abstract Harmonic Analysis, I-II, Springer-Verlag, 1963/1970.

[Hi-P] E. Hille and R.S. Phillips, Functional Analysis and Semi-Groups, Amer. Math. Soc. Colloquium Publ., 31 (1981).

[HJ] J. Hoffmann-Jørgensen, Weak compactness and tightness of subsets of $M(X)$, Math. Scand., 31 (1972), 127-150.

[Kad1] R.V. Kadison, Isometries of operator algebras, Ann. of Math., 54 (1951), 325-338.

[Kad2] _ Transformations of states in operator theory and dynamics, Topology, $\mathbf{3}$ (1965), 177-198.

[Kus] J. Kustermans, One-parameter representations on $C^{*}$-algebras, preprint (see funct - an/9707009).

[N] I. Namioka, Separate continuity and joint continuity, Pacific J. Math., 51 (1974), 515-531.

[P-S] A.L.T. Paterson and A.M. Sinclair, Characterization of isometries between $C^{*}$ algebras, J. London Math. Soc., 5(2) (1976), 755-761.

[Ped1] G.K. Pedersen, $C^{*}$-Algebras and their Automorphism Groups, Academic Press, 1979 .

[Ped2] $\quad S A W^{*}$-algebras and corona $C^{*}$-algebras, J. Operator Theory, 15 (1986), $15-32$.

[Rud] W. Rudin, Functional Analysis, McGraw-Hill, 1973.

[Sch] H.H. Schaefer, Topological Vector Spaces, Springer-Verlag, 1980.

[Stm] E. Størmer, On the Jordan structure of $C^{*}$-algebras, Trans. Amer. Math. Soc., 120 (1965), 438-447.

[S-Z] S.. Strătilă and L. Zsidó, Operator algebras, INCREST Prepublication, (19771979), 1-511.

[T1] D.C. Taylor, The strict topology for double centralizer algebras, Trans. Amer. math. Soc., 150 (1970), 633-643.

[T2] A general Phillips theorem for $C^{*}$-algebras and some applications, Pacific J. Math., 40 (1970), 477-488.

[Th] K. Thomsen, Jordan-morphisms in *-algebras, Proc. Amer. Math. Soc., 86 (1982), 283-286.

[WO] N.E. Wegge-Olsen, K-theory and $C^{*}$-algebras, Oxford University Press, 1993.

[Z1] L. Zsidó, Spectral and ergodic properties of the analytic generator, J. Approximation Theory, 20 (1977), 77-138.

[Z2] _ unpublished manuscript, 1979.

Received September 30, 1998. Both authors were Supported by M.U.R.S.T., C.N.R. and E.U. 
E-mail address: dantoni@axp.mat.uniroma2.it

UNIVERSITÀ DI ROMA "TOR VERGATA"

Via Della Ricerca Scientifica

00133 ROMA

ITALIA

E-mail address: zsido@axp.mat.uniroma2.it 